\title{
A Model for Internalized Stigma in Children and Adolescents with Epilepsy
}

\author{
Joan K. Austin, PhD, RN, FAAN ${ }^{\star}$, \\ Indiana University School of Nursing, Indianapolis, Indiana, U.S.A. \\ Susan M. Perkins, PhD, and \\ Department of Biostatistics, Indiana University School of Medicine, Indianapolis, Indiana, U.S.A. \\ David W. Dunn, MD \\ Departments of Psychiatry and Neurology, Indiana University School of Medicine, Indianapolis, \\ Indiana, U.S.A.
}

\section{Abstract}

Objective-Perceptions of stigma in children and adolescents with epilepsy are associated with higher rates of mental health problems. The purpose of this study was to test relationships in a model that identified variables most strongly associated with perceptions of stigma in children and adolescents with epilepsy. Our ultimate goal is to develop a theoretical foundation for future intervention research by identifying variables associated with perceptions of stigma that are potentially amenable to psychosocial interventions.

Methods-Participants were 173 children and adolescents with epilepsy who were between 9 and 14 years of age. Data were collected in telephone interviews. Stigma was measured using a self-report scale. Data were analyzed using structural equation modeling.

Results-Greater need for information and support, more fear and worry related to having epilepsy, greater seizure severity, and younger age were significantly associated with greater perceptions of stigma. Female gender, greater need for information and support, having at least one seizure in the past year, and lower self-efficacy for seizure management were significantly associated with more fear and worry related to having epilepsy.

Conclusions-Findings suggest that perceptions of stigma are associated with two variables that are amenable to psychosocial interventions: fear and worry about having epilepsy and need for information and support. Future research should test the efficacy of interventions that reduce fear and worry, provide information about epilepsy, and reduce need for support.

\section{(C) 2014 Elsevier Inc. All rights reserved.}

"Corresponding author; Joan K. Austin, PhD, RN, FAAN, Indiana University School of Nursing, 3040 N Ramble Road West, Bloomington, IN 47408, Tel: 812-332-8278, joausti@iu.edu.

Publisher's Disclaimer: This is a PDF file of an unedited manuscript that has been accepted for publication. As a service to our customers we are providing this early version of the manuscript. The manuscript will undergo copyediting, typesetting, and review of the resulting proof before it is published in its final citable form. Please note that during the production process errors may be discovered which could affect the content, and all legal disclaimers that apply to the journal pertain.

Conflict of Interest

Joan K. Austin and Susan M. Perkins report no conflicts of interest. David W. Dunn reports grant funding from Eli Lilly and Company. 


\section{Keywords}

epilepsy; stigma; children and adolescents; information needs; emotional support needs

\subsection{Introduction}

Stigma has been associated with epilepsy since ancient times. Goffman (1) describes stigma as status loss or discrediting caused by having a characteristic that is culturally defined as being different and undesirable. Weiss and Ramakrishna defined stigma that relates to a health condition as (2) "a social process or related personal experience characterized by exclusion, rejection, blame, or devaluation that results from experience or reasonable anticipation of an adverse social judgment about a person or group identified with a particular health problem" (p.536). In this definition perceptions of stigma can result either from personal experience of having been adversely judged or from the reasonable anticipation of being adversely judged because of the health condition. This definition emphasizes the importance of the stigma perceptions experienced by the individual with the health condition.

Stigma perceptions were conceptualized to occur at three different levels in a qualitative study of families of persons with mental illness: internalized, interpersonal, and institutional (3). Internalized stigma is felt within the person with the condition and reflects feelings, thoughts, beliefs, and fears related to being different. Interpersonal stigma occurs in interactions with others in which the person with the illness is treated differently and negatively because of the health condition. Institutionalized stigma reflects indirect expressions of differential treatment of persons with an illness as a group in the larger society. The focus of the model tested in this study was internalized stigma.

\subsection{Stigma in the Social Environments of Adolescents}

The social environment of children and adolescents with epilepsy may be difficult because their peers lack familiarity and knowledge about epilepsy. An Epilepsy Foundation survey of over 19,000 general population teens indicated that many of them held negative perceptions about people with epilepsy, which were most likely a result of lack of familiarity and knowledge about epilepsy (4). For example, in this survey 52\% of teens had never heard of epilepsy, $46 \%$ were not sure if epilepsy was contagious, $40 \%$ were not sure if people with epilepsy were dangerous, and 19\% thought that epilepsy was a form of mental illness. Other findings suggested social avoidance of persons with epilepsy. For example, only $31 \%$ reported that they would date someone with epilepsy. The rest (69\%) either responded that they were unsure or that they would not date someone with epilepsy. Another finding that suggests that social relationships might be difficult for teens with epilepsy was that $75 \%$ thought teens with epilepsy might be more likely to be bullied or picked on than their healthy peers.

A Canadian survey of 41 healthy teens and 118 chronically ill teens sought to determine if there was greater prejudice toward epilepsy than toward other chronic conditions. Results indicated that teens who had epilepsy rated epilepsy as having less of a social impact than 
did their peers. However, the total sample of both healthy and chronically ill teens rated epilepsy as being more likely to cause mental handicap, to lead to self-injury and death, and to cause injury to others than other chronic conditions (5). In this study the social impact of epilepsy was rated to be less than that for Down syndrome, similar to that for HIV infection and leukemia, and greater than that for asthma, diabetes, arthritis, or migraine. Comparisons with other chronic conditions also showed that people with epilepsy were perceived to have more behavior problems and to be less honest, less popular, worse at sports, less compassionate, and less fun. Another finding in this study indicated that some social avoidance of teens with epilepsy might be a result of lack of knowledge about how to handle a seizure if it should occur. Reasons teens gave for their unwillingness to befriend their peers with epilepsy were: concern about not knowing how to handle a seizure should it occur, difficulty in interacting with persons with epilepsy, concern about epilepsy being contagious, and concern about being responsible if a seizure or accident should occur (5). If children with epilepsy experience social encounters in which their peers treat them differently, they might be more likely to experience internalized stigma.

Both of these surveys suggest that lack of accurate information and misperceptions about epilepsy most likely contribute to the teen's negative beliefs about people with epilepsy. Lack of knowledge about epilepsy has been found to be associated with increased perceptions of stigma associated with epilepsy in the general public (6). Children and adolescents with epilepsy may be similar to their general population peers in lacking knowledge about epilepsy. Previous research has shown that children and adolescents with epilepsy desire more information about epilepsy (7) and want emotional support from others to help them cope with epilepsy (8). If lack of knowledge is associated with greater perceptions of stigma in the general population, it raises the question about how lack of knowledge about epilepsy in children and adolescents with epilepsy might be associated with their perceptions of stigma. It would be anticipated that lack of information about epilepsy would be associated with greater perceptions of internalized stigma in teens with epilepsy.

\subsection{Importance of Stigma Perceptions in Children and Adolescents with Epilepsy}

Studies show that when children and adolescents with epilepsy experience internalized stigma, they are more likely to have psychological problems. For example, in adolescents with idiopathic epilepsy, perceived stigma was significantly associated with lower selfesteem (9). In another study of children and adolescents with epilepsy, perceived stigma was associated with poorer self-concept and more depression symptoms (10). A third study found adolescents' feelings of stigma to be associated with increased anxiety and depression (11). In addition, parental perceptions that others had behaviors toward their child or others with epilepsy that reflected stigma were associated with increased depressed mood and behavior problems in their child as rated at home and at school (12). These studies suggest that it is important to identify factors that are associated with perceptions of stigma in children and adolescents with epilepsy in order to develop interventions that will reduce perceptions of stigma. 


\subsection{Stigma Model}

Our research team developed a model for internalized stigma that builds on our previous work on children's attitude toward having epilepsy (13). We used our attitude model as a foundation because we found perceptions of stigma to have a moderately strong positive correlation with negative attitudes toward having epilepsy $(r=.60)(10)$. In addition, we found both negative attitudes toward epilepsy (14) and higher levels of stigma perceptions (10) to be associated with more depression symptoms in adolescents with epilepsy. Key variables from our attitude model that were included in the stigma model were: fear and worry related to having epilepsy, family mastery, self-efficacy for managing seizures, and need for information and support related to having epilepsy. Other variables that were included based on prior research were: age, gender, age-of-onset, seizure severity, and having a seizure in the past year (13). Specifically, we anticipated that the following variables would be associated with greater perceptions of stigma: greater fear and worry related to having epilepsy, lower family mastery, lower self-efficacy for seizure management, and greater need for information and support. We also anticipated that younger age, female gender, earlier age of seizure onset, a seizure in the past year, and more severe seizures would be associated with greater perceptions of stigma.

\subsection{Methods}

\subsection{Subjects}

Baseline data from a larger longitudinal study of behavior problems in children and adolescents with epilepsy were used for this study. In the sample there were 173 children and adolescents who had been treated for epilepsy for at least 6 months. Of the 173 participants 171 were mothers $(98.84 \%)$ and the other 2 were stepmothers. The 85 girls and 88 boys were ages 9 through 14 years; $91 \%$ were Caucasian. Children were excluded from the sample if they had intellectual disability, a progressive brain disorder, or another chronic disorder. The mean age of seizure onset was 6.6 years and the average duration of epilepsy was 5.2 years. The majority of the children (69\%) had had at least one seizure in the past year and $79.8 \%$ had only one seizure type. Percentages of the primary seizure type were absence, $17.9 \%$; atonic, akinetic, or motor, $1.2 \%$; generalized tonic clonic, 19.7\%; elementary partial, $6.9 \%$; complex partial, $32.9 \%$; partial with secondary generalization, 19.7\%; and unclassified, $1.7 \%$.

\subsection{Measurement}

Factors explored for relationships with stigma perceptions were child characteristics, seizure variables, mastery in the family environment, child need for information and support, child fear and worry about epilepsy, and child self-efficacy for seizure management. Data were collected from the children and the primary caregiver, who was almost always the mother.

2.3.1 Stigma-The dependent variable, perceived stigma, was measured using the Child Stigma Scale (10), an 8-item scale on which children respond with how often they had feelings related to internalized stigma (e.g., feeling different, being embarrassed about 
having seizures, and avoiding disclosure). Past research has demonstrated that this scale has strong psychometric properties with this population (10).

2.3.2 Demographic and Seizure Variables-Child demographic characteristics measured were age and gender. Epilepsy variables investigated were age of onset of seizures, seizure in the past year (yes or no), and seizure severity. Seizure severity was measured by revising a scale developed for adults (15). Items reflected the intrusiveness, disruptiveness, and effects of the typical seizure. For example, on this scale tonic clonic seizures and secondary generalized seizures received a higher score than partial or absence seizures. Parents completed this scale.

2.3.3 Need for Information and Support-Need for information and support related to having epilepsy was measured using the Child Report of Psychosocial Care scale $(7,16)$. Content was developed based on interviews with children in which they were asked about the information they needed and what kind of help that they needed to help them deal with having epilepsy. This instrument has 12 items that ask about the extent ( $1=$ not very much, $2=$ some, $3=\mathrm{a}$ lot) of the need for information or support related to having epilepsy. The kind of information measured on this scale included need to learn more about causes of seizures, medication, and activity limitations. The type of support measured on this scale primarily reflects need for emotional support. Sample items include need to talk about their feelings about having epilepsy, to discuss their concerns and fears, and to get help with handling seizures at school. A higher total score reflects greater need for information and support.

2.3.4 Fear and Worry-Child fear and worry was measured using items from the Concerns and Fears About their Condition scale, which lists the most common fears and worries of children from our past research (17). Content for the scale was developed through open-ended interviews with children (17). Items included particular aspects of having epilepsy (e.g., worry about having another seizure, being injured during a seizure, and being sick). For this study items related to stigma worries were excluded from the list of child worries. Children and adolescents rated 9 items on 5-point scales of 1 (never) to 5 (always) with a higher total score indicating greater fear and worry.

2.3.5 Self-Efficacy-Self-Efficacy related to handling seizures was measured using the Seizure Self-Efficacy for Scale for Children (18). This is a 15-item scale on which children and adolescents rate the extent of their confidence that they can handle different aspects of handling seizures from 1 (I'm very unsure I can do that) to 5 (I am very sure I can do that). A higher total score reflects greater self-efficacy for managing their seizure condition. Prior research on this instrument has provided empirical support for psychometric properties with this population (18).

2.3.6 Mastery-Caregivers completed the instrument on the family environment. Mastery in the family environment was measured using caregivers' ratings on the mastery and health subscale of the Family Inventory of Resources for Management (19). A higher score reflects higher family functioning and support in the family environment. When this scale was used 
in prior research with this population, it was found to have good psychometric properties (20).

\subsection{Data Analyses}

Basic descriptive statistics were first generated for the sample. Structural equation modeling was used to assess the fit of the models described below. This procedure simultaneously assesses all paths in a model and provides global indices of fit. Our modeling strategy closely followed the modeling strategy used to develop Austin et al.'s Child Attitude model (13). After fitting the preliminary child attitude model using Stigma rather than Child Attitude as the primary outcome variable, we then considered the bivariate correlations and constructed a revised model that removed the non-significant paths from the preliminary model and added paths indicated by significant bivariate correlations. Of special interest were the proposed mediating effects of Fear and Worry and Self-Efficacy on the relationship between Information and Support or seizure condition variables and Stigma. To test these relationships, we performed the following steps as described in Baron and Kenny (21): (a) explored the bivariate correlations between Need for Information and Support and seizure condition variables, respectively, and Stigma; (b) used path model results to confirm the respective relationships of Need for Information and Support and seizure condition variables to Fear and Worry and Self-efficacy and of Fear and Worry and Self-Efficacy to Stigma; and (c) fit additional path models that included a direct effect for any psychosocial or seizure condition variables that met criteria a) and b) on Stigma as well as indirect paths through Fear and Worry and Self-efficacy.

Global indices of fit are reported for the final model obtained after assessing mediation. We report the $\chi^{2}$ value, Goodness of Fit Index (GFI), Comparative Index of Fit (CFI), Root Mean Square Error of Approximation (RMSEA), and Standardized Root Mean Square Residual (SRMR). LISREL was used to fit all models (22). Of the 173 children, 92\% ( $\mathrm{n}=$ 160) had complete data (i.e., no missing values) on all 10 variables of interest. The data for these 160 children were used in modeling and examining bivariate correlations. Using chisquare and two-sample t-tests, we found no differences in any study variables between those with and without complete data.

For all analyses, p-values $<0.05$ were considered statistically significant. In addition to LISREL, SAS Version 9.3 (Cary, NC) was used for statistical analyses.

\subsection{Results}

The mean Stigma score was 2.24 on a 1 to 5 scale, indicating that the average response to stigma items fell between "not often" and "sometimes." The mean score for items on the Fear and Worry scale was 2.61 on a 1 to 5 scale, which reflected an average response between "not often" and "sometimes." The mean score for Self-Efficacy for seizure management was 4.15 on a 1 to 5 scale, which fell closest to the response of "feeling a little sure" about their ability to handle their seizures. The mean score of Need for Information and Support was 1.83 on a 1 to 3 scale, which fell closest to the response of having "some" need. 
The final results of the model-fitting procedure are described in the Figure. Goodness-of-fit indices for this include a $\chi^{2}$ value of 15.71 ( $p$-value=.40), GFI of .98, CFI of 1.00, RMSEA of .01 (90\% CI .00 to .08), and SRMR of .04. Each of these indices indicates an acceptable fit. Fear and Worry was directly related to Stigma with greater Fear and Worry associated with higher levels of Stigma, but unlike the Child Attitude model, Self-Efficacy was not directly related to Stigma, but was related to Fear and Worry only with lower Self-Efficacy associated with higher levels of Fear and Worry. Of the four psychosocial and seizure condition variables (Need for Information and Support, Age of Onset, Seizure in the Past Year, and Seizure Severity), only Need for Information and Support was related to Fear and Worry, Self-Efficacy, and Stigma with greater Need for Information and Support associated with higher Fear and Worry, lower Self-Efficacy, and higher levels of Stigma). Age of Onset and Seizure Severity were not related to Fear and Worry or Self-Efficacy, and Age of Onset and Seizure Status were not related to Stigma. Seizure Status was also not related to SelfEfficacy. In addition, Family Mastery was not related to Fear and Worry, Self-Efficacy, or Stigma. Finally, Gender and Seizure in the past year were related to Fear and Worry only, and Age of Child and Seizure Severity to Stigma only. Female Gender and having a Seizure in the past year were associated with higher Fear and Worry, and younger Age and higher Seizure Severity were associated with higher levels of Stigma. With regard to mediation of Need for Information and Support by Fear and Worry and Stigma, if a direct effect of Need for Information and Support on Stigma was added to the model, this direct effect was statistically significant $(p$-value $<.0001)$. This indicates that Need for Information and Support had both direct effects on Stigma perceptions and indirect effects through SelfEfficacy and Fear and Worry. Again, this is different from the findings for the Child Attitude model, in which the direct effect of Need for Information and Support on Child Attitude was not significant.

\subsection{Discussion}

The purpose of this study was to test an internalized stigma model and identify factors that were the most strongly associated with perceptions of stigma in children and adolescents with epilepsy. Four variables were directly related to child perceptions of stigma (see Figure). Greater need for information and support, greater child fear and worry about seizures, more severe seizures, and younger child age were associated with higher levels of stigma perceptions. Predicted relationships with stigma that were not found to be directly associated with stigma were female gender, age of seizure onset, seizure in the past year, and self-efficacy for seizure management. However, these variables along with need for information and support were associated with more child fear and worry and thus were indirectly associated with perceived stigma. Finally, greater need for information and support was associated with poorer self-efficacy for seizure management, which made it the key predictor variable in the model.

Our findings provide a foundation for the development of interventions to address internalized or felt stigma in the clinical setting. Two key variables associated with greater stigma perceptions are potentially amenable to clinical intervention: need for information and support and child fear and worry related to having epilepsy. Another outcome of this study was that findings help identify that children who are most at risk for internalized 
stigma - those whose seizures are less well controlled, those who are fearful and worried, and those who need more information and support related to having epilepsy.

\subsubsection{Foundation for Psychosocial Interventions}

Few studies have focused on reducing internalized stigma in children and adolescents with epilepsy (23). Our findings strongly suggest that interventions to address perceived stigma in the clinical setting should include information about epilepsy and strategies for seeking support, which referred to primarily emotional support in this study. This variable had the strongest association with stigma and with child fear and worry in this study. Moreover, need for information and support was the only variable that was significantly associated with self-efficacy for seizure management. Although self-efficacy related to seizure management was not associated with perceived stigma in this study, prior research has demonstrated that lower self-efficacy was associated with greater perceptions of stigma (24). The strong associations between greater need for information and support and both greater fear and worry about having epilepsy and greater stigma perceptions suggest that both fear and worry and stigma might be addressed through meeting the children's needs for information and emotional support. The provision of education about epilepsy using either an animated video or an educational drama has been shown to improve knowledge about epilepsy and reduce stigma associated with epilepsy in general population children in the Czech Republic (25).

These findings suggest that interventions that help children address their specific needs for information about epilepsy might be helpful in reducing both fear and worry and stigma perceptions. A past study indicated that specific educational programs are needed for children because children do not appear to get their needs for information and support met from clinical care as usual. In this two-year longitudinal study of information and support needs of children with new onset seizures, Shore and colleagues (26) found that need for information and support remained high at 24 months after seizure onset. Examples of information needs were: $64 \%$ of children needed information on causes of seizures, $56 \%$ needed information on how to handle future seizures, and $62 \%$ needed information about keeping safe during a seizure. Examples of emotional support needs were: $64 \%$ wanted to talk with another child who had epilepsy, $49 \%$ wanted to talk to someone about handling seizures at school, and $47 \%$ wanted to talk to some about their future.

The finding that children and adolescents with epilepsy desire more information about epilepsy is similar to findings of a report by the Institute of Medicine (IOM) on epilepsy, Epilepsy Across the Spectrum: Promoting Health and Understanding (27). In the report it was emphasized that all people with epilepsy should receive information in writing about their particular epilepsy including specific details on type and on treatment. Other information that was recommended to be routinely provided to meet educational needs included issues related to reducing safety and mortality risks, strategies for living a healthy lifestyle, handling social relationships, emotional coping responses, and identifying available resources in the community. Additional areas of education for children and adolescents and their parents related to handling seizures at school, common mental health problems, helping children deal with fears and worry, and transition to adulthood and future career opportunities. 
The support needs assessed in this study were related to opportunities to discuss their future, to talk to other children who had epilepsy, to get help with how to tell others at school about the epilepsy, to discuss their fears and concerns, and to talk about feelings about having epilepsy. Some of the ways that these needs might be addressed are through receiving counseling, learning coping skills, engaging in conversations with other children who have epilepsy, and through family support groups (26). Our findings are consistent with a qualitative study of the lived experience adolescents with epilepsy (8). In that study the largest amount of time during the interviews focused on finding support and help from others in their environments. These findings indicate that interventions to reduce stigma will need to provide help with the development of skills to learn information and receive support related to epilepsy.

Child fear and worry also had a significant association with stigma perceptions. Based on this finding, one strategy for addressing internalized stigma in the clinical setting is to address children's and adolescents' worries related to having epilepsy. Children could be asked directly about what worries them about having seizures. Another approach would be to give children a short scale that lists the most common worries and ask them to circle those they were experiencing. In our early research we have found many children's worries to be based on inaccurate or incomplete information (17). For example, some children worried that they would keep getting sicker, would die, or would become mentally ill. These fears could be addressed by providing children and adolescents with age-appropriate accurate information about the actual likelihood of each of these events.

\subsubsection{Identification of Children and Adolescents at Risk for Stigma}

Our findings also help to identify children and adolescents who might be most vulnerable to feeling internalized stigma and thus are at greater risk for mental health problems. Younger age and more severe seizures (i.e., tonic clonic seizures or partial with secondary generalization) were significantly associated with stigma. Younger age being associated with greater perception of stigma is similar to a finding in a study of stigma in adolescents with epilepsy (9). A possible explanation for this finding is that younger children might be relatively less able to understand information about epilepsy that has been provided to them or they might be less able to cope with having seizures. Another possible explanation is that it might reflect what younger children have been told either directly or indirectly by adults in their home, school, and health care environments. If support is found for this latter explanation, interventions to reduce stigma might be needed for families, school, and health care settings.

Two additional variables were indirectly related to stigma through their association with fear and worry: female gender and a seizure in the past year. The associations of stigma with having a seizure in the past year and with having more severe seizures are similar to results by in a study of adults with epilepsy (28). Findings related to female gender are consistent with a prior study of attitudes in adolescents with epilepsy that found that girls had more negative attitudes toward having epilepsy than boys (29). These findings will help identify children and adolescents who should be targeted for assessment of stigma in the clinical setting. 


\subsubsection{Study Limitations}

Certain limitations of the study, especially the cross-sectional design, will influence interpretation of the findings. Most importantly, some of the relationships among model variables might be reciprocal. For example, being concerned about stigma could lead to more fear and worry and greater need for support as well as vice versa. In general, scores on the scales for measuring stigma and fear and worry were relatively low in this study; these findings might reflect that as a group this sample perceived relatively low levels of stigma as well as fear and worry. These findings also might indicate that the scales lack sensitivity. Future research is needed to further develop these scales.

\subsubsection{Conclusions and Future Research}

Perceptions of stigma are associated with two variables that are potentially amenable to psychosocial interventions: fear and worry about having epilepsy and need for information and support. Future research should test the efficacy of interventions that reduce fear and worry, provide information about epilepsy, and help children better meet their needs for support. In addition, research is needed to determine if addressing need for information and support in the clinical setting leads to less child fear and worry and greater child selfefficacy to manage their seizures.

\section{Acknowledgments}

Research on the stigma model was supported by grant R01 NR04536 from the National Institute of Nursing Research to J.K.A.

\section{References}

1. Goffman, E. Stigma: Notes on the management of spoiled identity. Prentice-Hall, Inc; Englewood Cliffs, N.J.: 1963.

2. Weiss MG, Ramakrishna J. Stigma interventions and research for international health. Lancet. Feb 11; 2006 367(9509):536-8. PubMed PMID: 16473134. [PubMed: 16473134]

3. Muhlbauer S. Experience of stigma by families with mentally ill members. Journal of the American Psychiatric Nurses Association. 2002; 8:76-83.

4. Austin JK, Shafer PO, Deering JB. Epilepsy familiarity, knowledge, and perceptions of stigma: Report from a survey of adolescents in the general population. Epilepsy \& Behavior. 2002; 3:36875. [PubMed: 12609335]

5. Cheung C, Wirrell E. Adolescents' perception of epilepsy compared with other chronic diseases: "through a teenager's eyes". Journal of child neurology. Mar; 2006 21(3):214-22. PubMed PMID: 16901423. [PubMed: 16901423]

6. Diiorio C, Kobau R, Holden EW, Berkowitz JM, Kamin SL, Antonak RF, et al. Developing a measure to assess attitudes toward epilepsy in the US population. Epilepsy \& behavior: E\&B. Dec; 2004 5(6):965-75. PubMed PMID: 15582846.

7. McNelis A, Musick B, Austin JK, Dunn D, Creasy K. Psychosocial care needs of children with new-onset seizures. 2. The Journal of neuroscience nursing: journal of the American Association of Neuroscience Nurses. Jun; 1998 30(3):161-5. PubMed PMID: 9689607. [PubMed: 9689607]

8. Eklund P, Sivberg B. Adolescents' lived experience of epilepsy. Journal of Neuroscience Nursing. Feb; 2003 35(1):40-9. PubMed PMID: 12789720.eng. [PubMed: 12789720]

9. Westbrook LE, Bauman LJ, Shinnar S. Applying stigma theory to epilepsy: a test of a conceptual model. Journal of pediatric psychology. Oct; 1992 17(5):633-49. PubMed PMID: 1432485.

[PubMed: 1432485] 
10. Austin JK, MacLeod J, Dunn DW, Shen J, Perkins SM. Measuring stigma in children with epilepsy and their parents: instrument development and testing. Epilepsy \& behavior: E\&B. Aug; 2004 5(4):472-82. PubMed PMID: 15256183.

11. Adewuya AO, Ola BA. Prevalence of and risk factors for anxiety and depressive disorders in Nigerian adolescents with epilepsy. Epilepsy \& Behavior. 2005; 6:342-7. [PubMed: 15820341]

12. Carlton-Ford S, Miller R, Nealeigh N, Sanchez N. The effects of perceived stigma and psychological over-control on the behavioural problems of children with epilepsy. Seizure. 1997; 6:383-91. [PubMed: 9663802]

13. Austin JK, Dunn DW, Perkins SM, Shen J. Youth With Epilepsy: Development of a Model of Children's Attitudes Toward Their Condition. Children's health care: journal of the Association for the Care of Children's Health. 2006; 35(2):123-40. PubMed PMID: 17075611. Pubmed Central PMCID: 1627850.

14. Dunn DW, Austin JK, Huster GA. Symptoms of depression in adolescents with epilepsy. Journal of the American Academy of Child and Adolescent Psychiatry. 1999; 38:1132-8. [PubMed: 10504812]

15. Baker GA, Smith DF, Dewey M, Morrow J, Crawford PM, Chadwick DW. The development of a seizure severity scale as an outcome measure in epilepsy. Epilepsy Research. 1991; 8:245-51. [PubMed: 1868824]

16. Austin JK, Dunn DW, Huster G, Rose D. Development of scales to measure psychosocial care needs of children with seizures and their parents. 1. The Journal of neuroscience nursing: journal of the American Association of Neuroscience Nurses. Jun; 1998 30(3):155-60. PubMed PMID: 9689606. [PubMed: 9689606]

17. Austin JK. Concerns and fears of children with seizures. Clinical Nursing Practice in Epilepsy. 1993; 1(4):4-6. 10. [PubMed: 8156129]

18. Caplin D, Austin JK, Dunn DW, Shen J, Perkins S. Development of a self-efficacy scale for children and adolescents with epilepsy. Children's Health Care. 2002; 31(4):295-309.

19. McCubbin, HI.; Thompson, AI.; McCubbin, MA. Family assessment: REsiliency, copin and adaptation -- Inventories for research and practice. University of Wisconsin; Madison, Wisconsin: 1996.

20. Austin JK, Dunn DW, Johnson CS, Perkins SM. Behavioral issues involving children and adolescents with epilepsy and the impact of their families: recent research data. Epilepsy \& behavior: E\&B. Oct; 2004 5(Suppl 3):S33-41. PubMed PMID: 15351344.

21. Baron RM, Kenny DA. Moderator-mediator variable distinction in social psychological research: Conceptual, strategic, \& statistical considerations. Journal of Personality and Social Psychology. 1986; 51(6):1173-82. [PubMed: 3806354]

22. Joreskog, KG.; Sorbom, D. LISREL 8: User's reference guide. Scientific Software International; Chicago: 1996.

23. Birbeck G. Interventions to reduce epilepsy-associated stigma. Psychology, health \& medicine. Aug; 2006 11(3):364-6. PubMed PMID: 17130072.

24. Smith G, Ferguson PL, Saunders LL, Wagner JL, Wannamaker BB, Selassie AW. Psychosocial factors associated with stigma in adults with epilepsy. Epilepsy \& behavior: E\&B. Nov; 2009 16(3):484-90. PubMed PMID: 19782005.

25. Brabcova D, Lovasova V, Kohout J, Zarubova J, Komarek V. Improving the knowledge of epilepsy and reducing epilepsy-related stigma among children using educational video and educational drama--a comparison of the effectiveness of both interventions. Seizure: the journal of the British Epilepsy Association. Apr; 2013 22(3):179-84. PubMed PMID: 23265921. [PubMed: 23265921]

26. Shore CP, Buelow JM, Austin JK, Johnson CS. Continuing psychosocial care needs in children with new-onset epilepsy and their parents. The Journal of neuroscience nursing: journal of the American Association of Neuroscience Nurses. Oct; 2009 41(5):244-50. PubMed PMID: 19835237. Pubmed Central PMCID: 2864533. [PubMed: 19835237]

27. IOM. Epilepsy across the spectrum: Promoting health and understanding. The National Academies Press; Washington, D.C.: 2012. p. 537 
28. DiIorio C, Osborne Shafer P, Letz R, Henry T, Schomer DL, Yeager K, et al. The association of stigma with self-management and perceptions of health care among adults with epilepsy. Epilepsy \& behavior: E\&B. Jun; 2003 4(3):259-67. PubMed PMID: 12791327.

29. Heimlich TE, Westbrook LE, Austin JK, Cramer JA, Devinsky O. Brief report: Adolescents' attitudes toward epilepsy: Further validation of the Child Attitude Toward Illness Scale (CATIS). Journal of pediatric psychology. 2000; 25(5):339-45. [PubMed: 10880064] 


\section{Highlights}

- We developed a model for felt stigma in children with epilepsy

- We identified psychosocial variables amenable to interventions

- Interventions should include education and coping skills training 


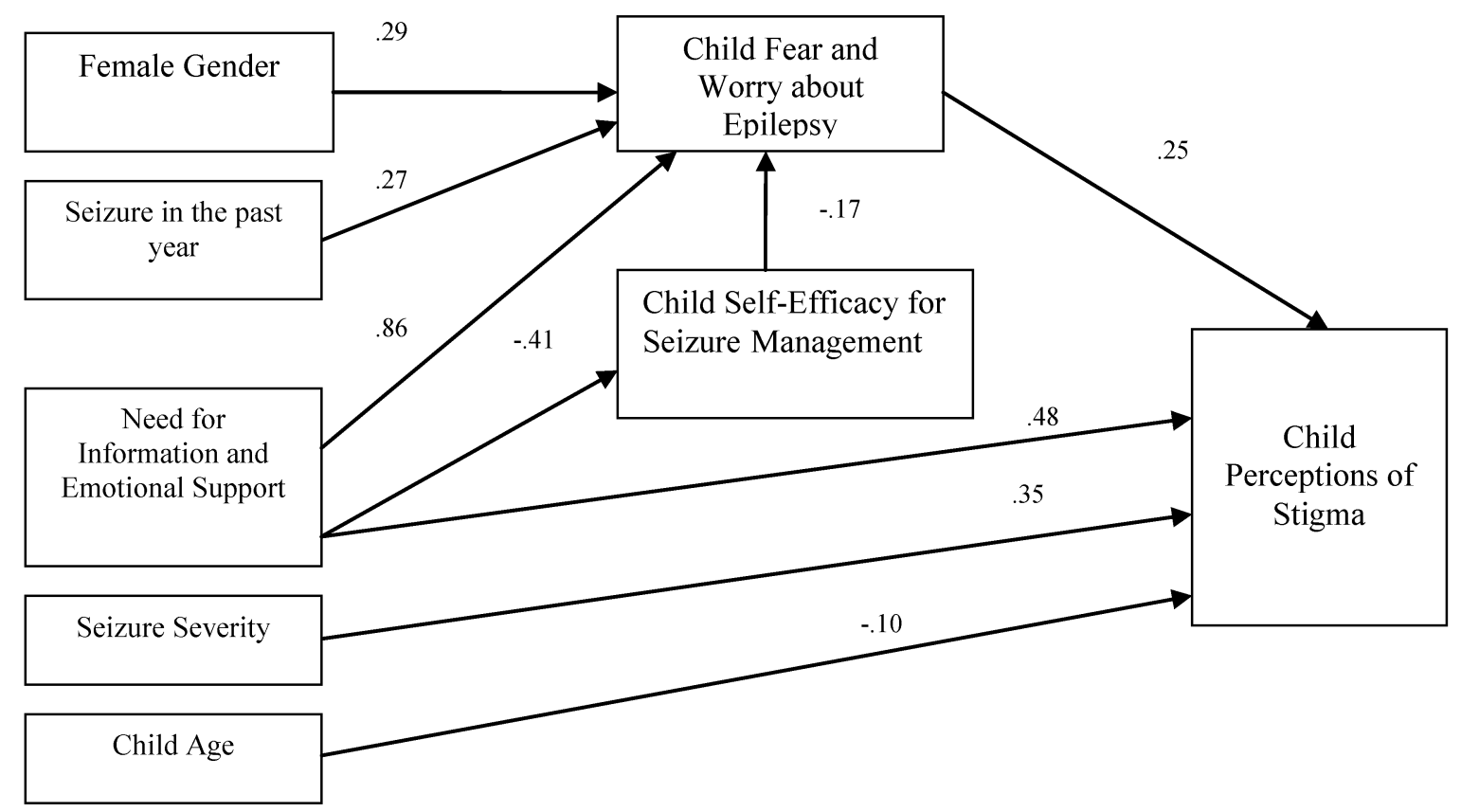

Figure 1.

Relationships among Variables in Internalized Stigma Model 